\title{
Genome-wide association analysis reveals loci and candidate genes involved in fiber quality traits in sea island cotton (Gossypium barbadense)
}

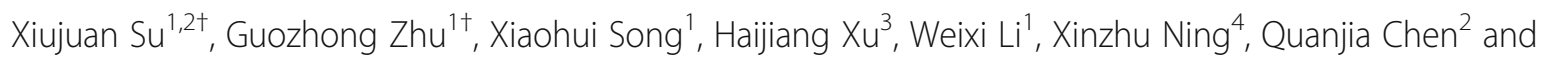
Wangzhen Guo ${ }^{1 *}$ (D)

\begin{abstract}
Background: Sea island cotton (Gossypium barbadense) has markedly superior high quality fibers, which plays an important role in the textile industry and acts as a donor for upland cotton (G. hirsutum) fiber quality improvement. The genetic characteristics analysis and the identification of key genes will be helpful to understand the mechanism of fiber development and breeding utilization in sea island cotton.

Results: In this study, 279 sea island cotton accessions were collected from different origins for genotyping and phenotyping fiber quality traits. A set of 6303 high quality single nucleotide polymorphisms (SNPs) were obtained by high-density CottonSNP80K array. The population characteristics showed that the sea island cotton accessions had wide genetic diversity and were clustered into three groups, with Group1 closely related to Menoufi, an original sea island cotton landrace, and Group2 and Group3 related to widely introduced accessions from Egypt, USA and Former Soviet Union. Further, we used 249 accessions and evaluated five fiber quality traits under normal and salt environments over 2 years. Except for fiber uniformity (FU), fiber length (FL) and fiber elongation (FE) were significantly decreased in salt conditions, while fiber strength (FS) and fiber micronaire (MIC) were increased. Based on 6303 SNPS and genome-wide association study (GWAS) analysis, a total of 34 stable quantitative trait loci (QTLs) were identified for the five fiber quality traits with 25 detected simultaneously under normal and salt environments. Gene Ontology (GO) analysis indicated that candidate genes in the 25 overlapped QTLs were enriched mostly in "cellular and biological process". In addition, "xylem development" and "response to hormone" pathways were also found. Haplotype analyses found that GB_A03G0335 encoding an E3 ubiquitin-protein ligase in QTL TM6004 had SNP variation (A/C) in gene region, was significantly correlated with $\mathrm{FL}, \mathrm{FS}, \mathrm{FU}$, and $\mathrm{FE}$, implying a crucial role in fiber quality.
\end{abstract}

Conclusions: The present study provides a foundation for genetic diversity of sea island cotton accessions and will contribute to fiber quality improvement in breeding practice.

Keywords: Gossypium barbadense, Genetic diversity, Genome-wide association study, Fiber quality, Quantitative trait loci, Salt stress

\footnotetext{
* Correspondence: moelab@njau.edu.cn

${ }^{+}$Xiujuan Su and Guozhong Zhu contributed equally to this work.

'State Key Laboratory of Crop Genetics and Germplasm Enhancement,

Engineering Research Center of Hybrid Cotton Development (the Ministry of

Education), Nanjing Agricultural University, Nanjing 210095, China

Full list of author information is available at the end of the article
}

(c) The Author(s). 2020 Open Access This article is licensed under a Creative Commons Attribution 4.0 International License, which permits use, sharing, adaptation, distribution and reproduction in any medium or format, as long as you give appropriate credit to the original author(s) and the source, provide a link to the Creative Commons licence, and indicate if changes were made. The images or other third party material in this article are included in the article's Creative Commons licence, unless indicated otherwise in a credit line to the material. If material is not included in the article's Creative Commons licence and your intended use is not permitted by statutory regulation or exceeds the permitted use, you will need to obtain permission directly from the copyright holder. To view a copy of this licence, visit http://creativecommons.org/licenses/by/4.0/ The Creative Commons Public Domain Dedication waiver (http://creativecommons.org/publicdomain/zero/1.0/) applies to the data made available in this article, unless otherwise stated in a credit line to the data. 


\section{Background}

Cotton (Gossypium spp.) is one of the most important crops producing natural fibers for the textile industry. Upland cotton (G. hirsutum) and sea island cotton (G. barbadense) are the two main cultivated tetraploid species accounting for more than $97 \%$ of the total cotton production [1]. Upland cotton produces high yields with moderate fiber quality, whereas sea island cotton has exceptionally high quality fibers [2]. As the textile industry rapidly develops and the demand for textile products continues to diversify, the improvement of cotton fiber quality is more and more important [3]. The introgression of chromosome segments can effectively combine the yield advantage of upland cotton with the quality improvement from sea island cotton [4]. Hence, it is of great significance to explore the elite chromosome segments in G. barbadense for improving cotton fiber quality in breeding.

Identifying the quantitative trait loci (QTLs) of the target traits and marker assisted selection (MAS) greatly accelerated the breeding of cotton varieties. With the development of molecular marker, SNP array and resequencing technology, a batch of QTLs for fiber yield, quality and salt tolerance of upland cotton have been explored. Using SSR markers, Shen et al. (2005) identified 39 QTLs associated with fiber quality traits of upland cotton [5]. Sun et al. (2018) genotyped 719 upland cotton germplasm accessions by an Illumina CottonSNP63K array and 62 SNP loci were significantly associated with yield-related traits [6]. Via specific-locus amplified fragment sequencing (SLAF-seq), Su et al. (2016) detected 12 SNPs associated with lint percentage of upland cotton in four different environments [7]. For salt tolerance, 8 and 23 SNPs were identified to be associated significantly with the salt-tolerance related traits $[8,9]$. These studies provided QTLs and gene resources for developing upland cotton varieties with high yields and salt stress tolerance.

Compared with G. hirsutum, less studies for genetic diversity and QTL mining related to target traits were reported using natural population in G. barbadense. In addition, Xinjiang is the main production area of sea island cotton in China, however, most of the cultivated land in Xinjiang is saline alkali land [10]. Salinity is a serious threat for fiber yield and quality [11]. In upland cotton, higher $\mathrm{Na}^{+}$levels in soil can decrease fiber length, strength, and micronaire [12]. However, the related report on sea island cotton is rare. Based on these, in this study, 279 sea island cotton accessions were collected from different origins, and genotyping for population structure and phenotyping for fiber qualities in different soil environments over 2 years were systematically investigated. By combining the two datasets, genome-wide association study (GWAS) analysis identified the stable QTLs and candidate genes associated with fiber qualities. The finding will provide elite genetic resources with candidate QTLs and genes for fiber quality improvement in cotton breeding.

\section{Results \\ Genetic variation based on SNPs}

The 279 sea-island cotton accessions (Additional file 1: Table S1) were genotyped using the CottonSNP80K array. The genotypic data revealed that these cotton accessions possessed a high average call rate of $96.54 \%$ (Additional file 2: Fig. S1). We further filtered some loci with call frequency < $80 \%$, minor allele frequency $(\mathrm{MAF})<0.01$ or mapping to multiple loci of chromosomes). As a result, a final set of 6303 SNPs were obtained, with 3656 and 2647 SNPs on the At and Dt sub-genomes, respectively (Additional file 3: Fig. S2). To evaluate the genotyping accuracy and reproducibility, eight cotton accessions from different sources were set biological replicates for genotyping analysis. The genotyping consistencies between the replicates were ranged from 86.3 to $99.8 \%$, indicating that there exists heterogeneity of the same accession with different sources. Of them, Hai7124 and Xinhai 26 from different sources were detected the highest consistency with 99.8 and 99.3\%, respectively. We also evaluated the polymorphism information content (PIC) values of 279 accessions, which varied from 0.189 to 0.469 among chromosomes, and the mean PIC value of the At and Dt sub-genomes was 0.391 and 0.338, respectively (Additional file 4: Table S2). These results showed that the CottonSNP80K array could genotype sea island cotton accessions with good efficiency and accuracy.

\section{Population characteristics analysis}

In order to explore the population characteristics of the sea island cotton accessions, a neighbor-joining tree was conducted using TASSEL 5.0. It showed that the 279 accessions could be clustered into three groups, which contained 87, 112 and 80 in Group1, Gourp2 and Group3, respectively (Fig. 1a). The 39 landraces, which were earlier introduced into China and contributed to sea island cotton breeding of China, were widely distributed in the three groups. Group1 contained three introduced accessions, Giza 36, Giza 81 and Menoufi, that they were all from Egypt and Menoufi was an original sea island cotton landrace. Group2 and Group3 were clustered with widely introduced accessions from Egypt, USA and Former Soviet Union, with 31 introduced accessions in Group2 and five in Group3. The PCA (Fig. 1b) and population structure analysis (Fig. 1c) also supported the 279 accessions could be clustered into three groups which agreed well with the neighbor-joining tree. We measured the genetic diversity of each group by calculating nucleotide diversity $(\pi)$ values (Fig. 1d), and found that Group1 had the highest genetic diversity $(\pi=0.35)$, while Group3 was the lowest $(\pi=$ 0.29 ). Further, population fixation statistics (Fst) indicated 


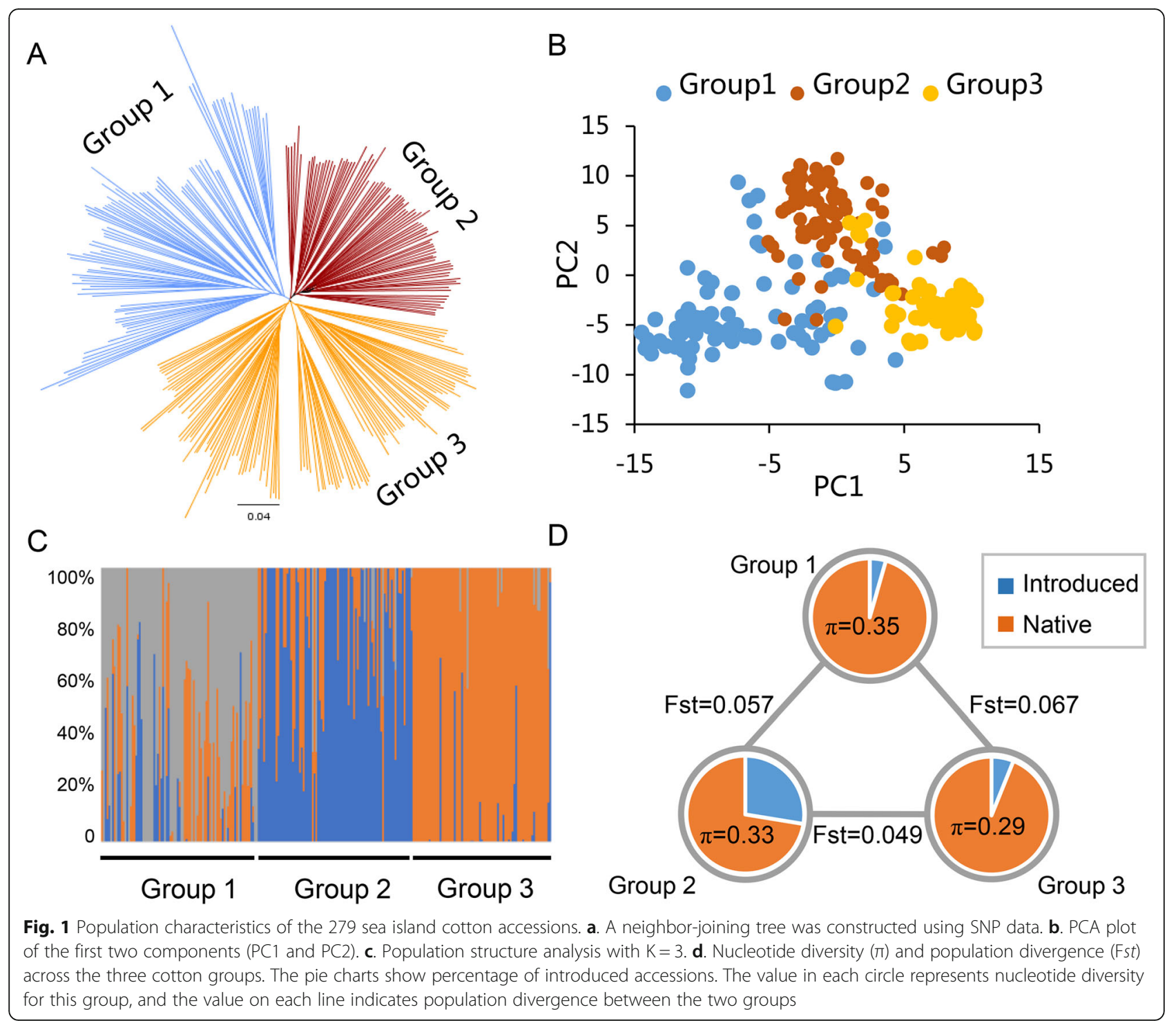

that the Fst value between Group1 and Group2 or between Group1 and Group3 was higher than that between Group2 and Group3 (Fig. 1d), implying that Group1 had the highest differentiation compared with the other two groups.

\section{Phenotypic variation in fiber quality traits}

To evaluate the phenotypic variation in fiber quality traits in the association population, we analyzed five traits in two environments over 2 years. The results showed the wide variation in the natural population with 29.35 to $39.68 \mathrm{~mm}$ for FL, 28.29 to $48.10 \mathrm{cN} /$ tex for FS, 3.10 to 5.23 for MIC, 81.75 to $88.65 \%$ for FU and 6.52 to $7.12 \%$ for FE, respectively (Table 1). MIC and FS had the largest coefficients of variation $(\mathrm{CV})$, ranging from 6.28 to $8.31 \%$ and from 8.53 to $10.91 \%$, respectively, followed by FL (4.27-5.35\%). FE and FU had the smallest $\mathrm{CV}$, ranging from 1.04 to $1.48 \%$ and from 0.93 to $1.06 \%$, respectively. Density distributions indicated that FL and FE had more high value distribution under normal conditions than under salt conditions. In details, the distribution of FL value higher than 37 and of $\mathrm{FE}$ value higher than 6.9 under normal conditions was more than that under salt conditions. For FS and MIC, it had higher value distribution under salt conditions than under normal conditions with greater than 32 and 4, respectively. For FU, the distribution was more concentrated under salt conditions than that under normal conditions (Additional file 5: Fig. S3). Paired-samples $t$-tests further showed that comparing to normal conditions, FL and FE decreased significantly with 0.46 and $1.01 \%$ under salt conditions, respectively, while FS and MIC increased with 4.73 and $1.74 \%$, respectively. There was no significant difference in FU in the two environments (Fig. 2). Correlation analysis showed high 
Table 1 Phenotypic variation for five fiber quality traits on 249 sea island cotton accessions

\begin{tabular}{|c|c|c|c|c|c|c|}
\hline Trait & Environment ${ }^{a}$ & Min & Max & Mean & SD & CV (\%) \\
\hline \multirow[t]{6}{*}{$\mathrm{FL}(\mathrm{mm})$} & FL17-C & 31.15 & 39.05 & 36.11 & 1.67 & 4.62 \\
\hline & FL17-S & 31.25 & 38.60 & 35.91 & 1.53 & 4.27 \\
\hline & FL18-C & 30.31 & 38.45 & 35.82 & 1.62 & 4.52 \\
\hline & FL18-S & 29.35 & 39.68 & 35.69 & 1.91 & 5.35 \\
\hline & FL-C-BLUP & 25.75 & 41.14 & 35.96 & 3.11 & 8.66 \\
\hline & FL-S-BLUP & 25.14 & 41.50 & 35.80 & 3.22 & 8.98 \\
\hline \multirow[t]{6}{*}{ FS (cN/tex) } & FS17-C & 29.47 & 45.47 & 37.23 & 3.72 & 10.00 \\
\hline & FS17-S & 30.90 & 46.30 & 38.07 & 3.25 & 8.53 \\
\hline & FS18-C & 28.29 & 48.10 & 37.03 & 4.04 & 10.91 \\
\hline & FS18-S & 31.47 & 47.20 & 39.70 & 3.81 & 9.61 \\
\hline & FS-C-BLUP & 21.31 & 54.81 & 37.13 & 7.28 & 19.61 \\
\hline & FS-S-BLUP & 23.92 & 51.46 & 38.89 & 6.59 & 16.96 \\
\hline \multirow[t]{6}{*}{ MIC } & MIC17-C & 3.38 & 4.99 & 4.19 & 0.27 & 6.51 \\
\hline & MIC17-S & 3.62 & 4.98 & 4.25 & 0.27 & 6.28 \\
\hline & MIC18-C & 3.11 & 5.12 & 4.02 & 0.33 & 8.31 \\
\hline & MIC18-S & 3.10 & 5.23 & 4.11 & 0.33 & 8.13 \\
\hline & MIC-C-BLUP & 2.69 & 5.74 & 4.11 & 0.53 & 12.87 \\
\hline & MIC-S-BLUP & 2.69 & 5.92 & 4.18 & 0.52 & 12.45 \\
\hline \multirow[t]{6}{*}{ FU (\%) } & FU17-C & 83.35 & 88.13 & 86.28 & 0.90 & 1.04 \\
\hline & FU17-S & 84.40 & 88.65 & 87.07 & 0.81 & 0.93 \\
\hline & FU18-C & 82.56 & 87.98 & 85.64 & 0.91 & 1.06 \\
\hline & FU18-S & 81.75 & 86.55 & 84.97 & 0.81 & 0.95 \\
\hline & FU-C-BLUP & 81.16 & 89.67 & 85.96 & 1.51 & 1.76 \\
\hline & FU-S-BLUP & 81.40 & 88.46 & 86.02 & 1.34 & 1.56 \\
\hline \multirow[t]{6}{*}{ FE (\%) } & FE17-C & 6.62 & 7.11 & 6.89 & 0.10 & 1.48 \\
\hline & FE17-S & 6.52 & 6.95 & 6.74 & 0.08 & 1.21 \\
\hline & FE18-C & 6.65 & 7.07 & 6.88 & 0.07 & 1.08 \\
\hline & FE18-S & 6.73 & 7.12 & 6.89 & 0.07 & 1.04 \\
\hline & FE-C-BLUP & 6.52 & 7.18 & 6.89 & 0.13 & 1.88 \\
\hline & FE-S-BLUP & 6.53 & 7.02 & 6.82 & 0.10 & 1.42 \\
\hline
\end{tabular}

${ }^{a} 17,18$ and BLUP indicated the phenotype data in 2017, 2018 and BLUP evaluated in the same environment, $C$ : normal condition; $\mathrm{S}$ : salt condition $F L$ fiber length, $F S$ fiber strength, MIC fiber micronaire, FU fiber uniformity, FE fiber elongation

phenotypic correlation for each trait under both normal and salt conditions. In addition, under the same condition, FL was significantly positively correlated with FS, FE and FU, and a positive correlation was also found among FS, FE and FU. MIC was significantly negatively correlated with FL and FS, and positively correlated with FE (Additional file 6: Fig. S4).

\section{Genome-wide association studies}

The GWAS was conducted for the five fiber quality traits with a multi-loci mixed linear model [13]. In total, 102 quantitative trait nucleotides (QTNs) on 26 chromosomes were identified as significantly associated with the traits (Additional file 7: Table S3). We define the flank $200 \mathrm{~Kb}$ regions of QTN as an initial QTL and merge the overlapped QTLs to obtain the final QTLs as described previously [14]. In total, 81 QTLs were detected 413 times under different environments and a best linear unbiased predictors (BLUP) by six multi-loci MLM models. Considering that it could be false positive in QTLs with less association frequency, we selected QTLs with more than three association times as stable QTLs for subsequent analysis (Additional file 8: Fig. S5). As a result, 34 stable QTLs were identified including nine high frequency associated QTLs with association times greater than 10 (Table 2 and Additional file 9: Table S4).

Due to the high correlation, the overlapped stable QTLs among the five traits were also detected (Additional file 9: Table S4). It showed that 18 stable QTLs for each of four traits (FL: 4, FS: 4, MIC: 5 and FE: 5) were specifically detected and 16 stable overlapped QTLs were detected in two or more traits. Of them, QTL TM6004 was detected to simultaneously associate with FL, FS, FE and FU, with the most association times of 75 . There were also four QTLs were detected to be associated with three traits (Additional file 9: Table S4). These stable QTLs were widely distributed on At and Dt subgenome with a bit more loci of FL, FS, FE and FU on Dt subgenome (Fig. 3a). Chromosome distribution showed that most QTLs for MIC and FE were located on A01 and D05, respectively (Fig. 3b). In different environments, many overlapped QTLs were also detected under both normal and salt conditions (74\% of total QTLs), especially for FL and FS with 64 and 55\% overlapped QTLs, respectively (Fig. 3c), implying the high and stable heritability for most of fiber quality traits.

\section{Identification on candidate genes in QTLs}

Potential candidate genes in these stable QTL regions were extracted based on the released G. barbadense Hai7124 genome [15]. In total, 998 candidate genes were identified in the stable QTL regions, with 302 of FL, 251 of FS, 373 of MIC, 84 of FU and 583 of FE (Additional file 10: Table S5). Considering the two environments, 118 and 95 genes were specifically identified associated with the fiber quality traits under normal and salt conditions, respectively, while 785 genes were simultaneously identified. GO analysis showed that the 95 genes specifically identified in salt conditions were enriched in "response to stimulus" $\left(P\right.$-value $\left.4.8 \times 10^{-5}\right)$, and the 118 genes specifically identified in normal conditions were mainly involved in "cellular process". We found the 785 candidate genes simultaneously identified under normal and salt conditions were enriched mostly in "cellular process" 

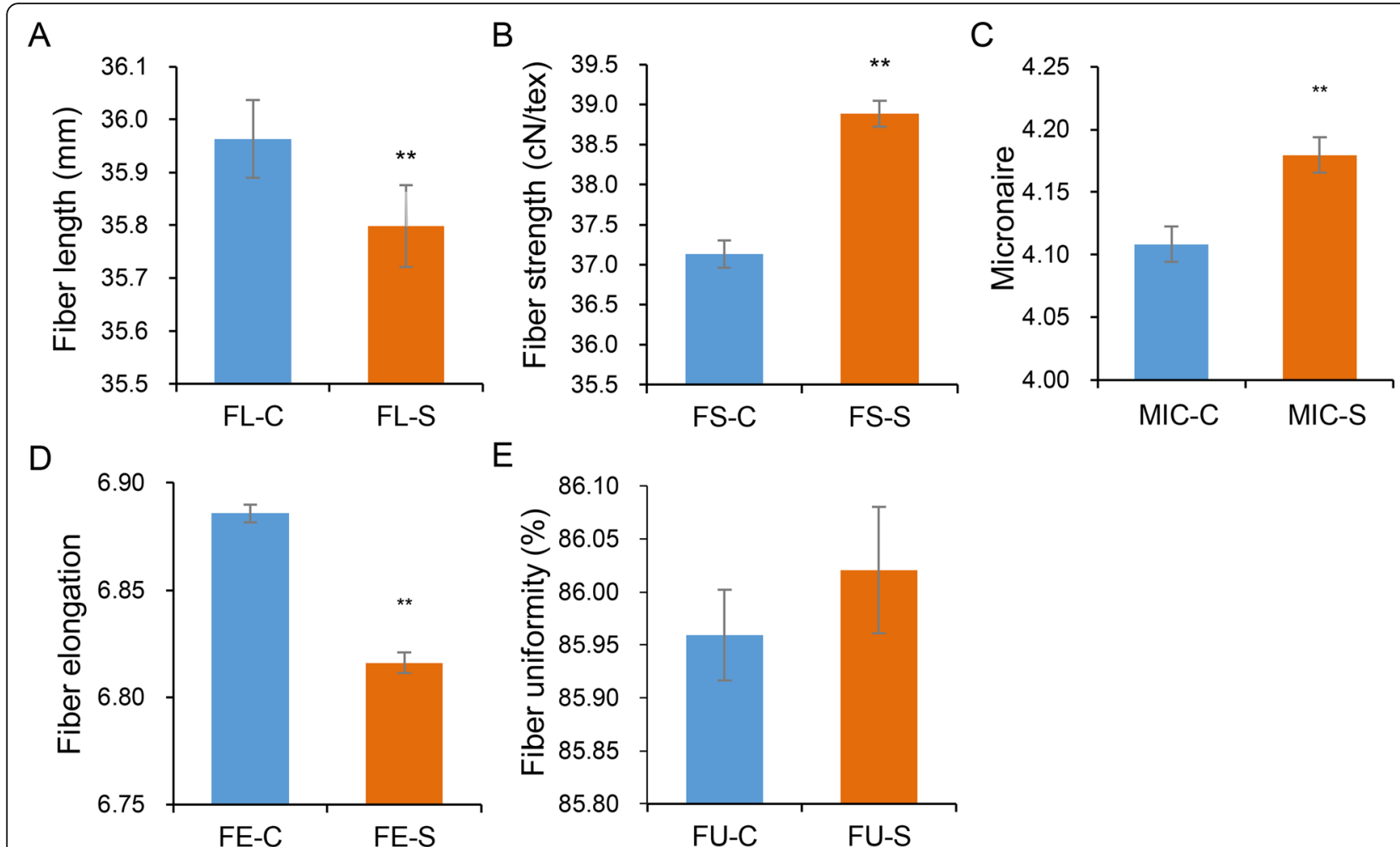

$\mathrm{E}$

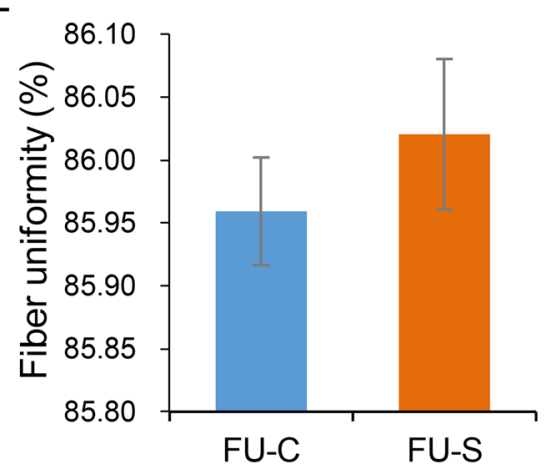

Fig. 2 Comparison of different phenotypic data under normal and salt environments. a. Fiber length (FL). b. fiber strength (FS). c. fiber micronaire (MIC) d. fiber elongation (FE). e. fiber uniformity (FU). Significant difference of single trait was calculated with paired-samples $t$-tests. ** indicates a significance level of 0.01

Table 2 Identification on stable QTL related to five fiber quality traits by multi-loci MLM model

\begin{tabular}{llll}
\hline Trait $^{\text {a }}$ & Environment & Number of QTL & QTL counts \\
\hline FL & Normal & 9 & 45 \\
& Salt & 9 & 41 \\
& Total & 11 & 86 \\
FS & Normal & 9 & 52 \\
& Salt & 8 & 49 \\
& Total & 11 & 101 \\
MIC & Normal & 10 & 35 \\
& Salt & 7 & 23 \\
& Total & 13 & 58 \\
FU & Normal & 2 & 12 \\
& Salt & 4 & 26 \\
& Total & 4 & 38 \\
FE & Normal & 10 & 36 \\
& Salt & 11 & 28 \\
& Total & 17 & 64 \\
Total & & 34 & 347 \\
\hline FL fiber & & FS fiber stength &
\end{tabular}

${ }_{\mathrm{a}}^{\mathrm{F} L}$ fiber length, FS fiber strength, MIC fiber micronaire, FU fiber uniformity, FE fiber elongation and "biological process". Also, "xylem development", "response to hormone" and "regulation of post-embryonic development" were also detected (Additional file 11: Table S6). According to tissues expression profile, we further identified 572 fiber expressed genes in these stable QTL regions, indicating they play roles in fiber development (Additional file 12: Table S7).

\section{Mining of key genes for fiber quality traits}

We further analyzed the nine high frequency associated QTLs for mining key candidate genes. The QTL TM6004, which had a QTN at 4,186,816 bp of the QTL region located on A03: 3,986,816 bp - 4,386,816 bp (Fig. 4a), was simultaneously associated with FE, FL, FS and FU with high frequency detected times, and explained more than $19.9 \%$ of the variation in fiber quality traits both in the normal and salt environments. Sequence analysis showed that the QTN of TM6004 (from $A$ to $C$ ) was located in the intron region of the gene GB_A03G0335 which encodes an E3 ubiquitin-protein ligase. Through a Student's $t$ test, we found that the FL, FS, FU and FE increased respectively by 6.9, 22.5, 1.5 and $1.2 \%$ with the $\mathrm{C}$ haplotype comparing with the $\mathrm{A}$ haplotype (Fig. 4b), implying that QTN TM6004 might play important roles in fiber development. Beside this, 


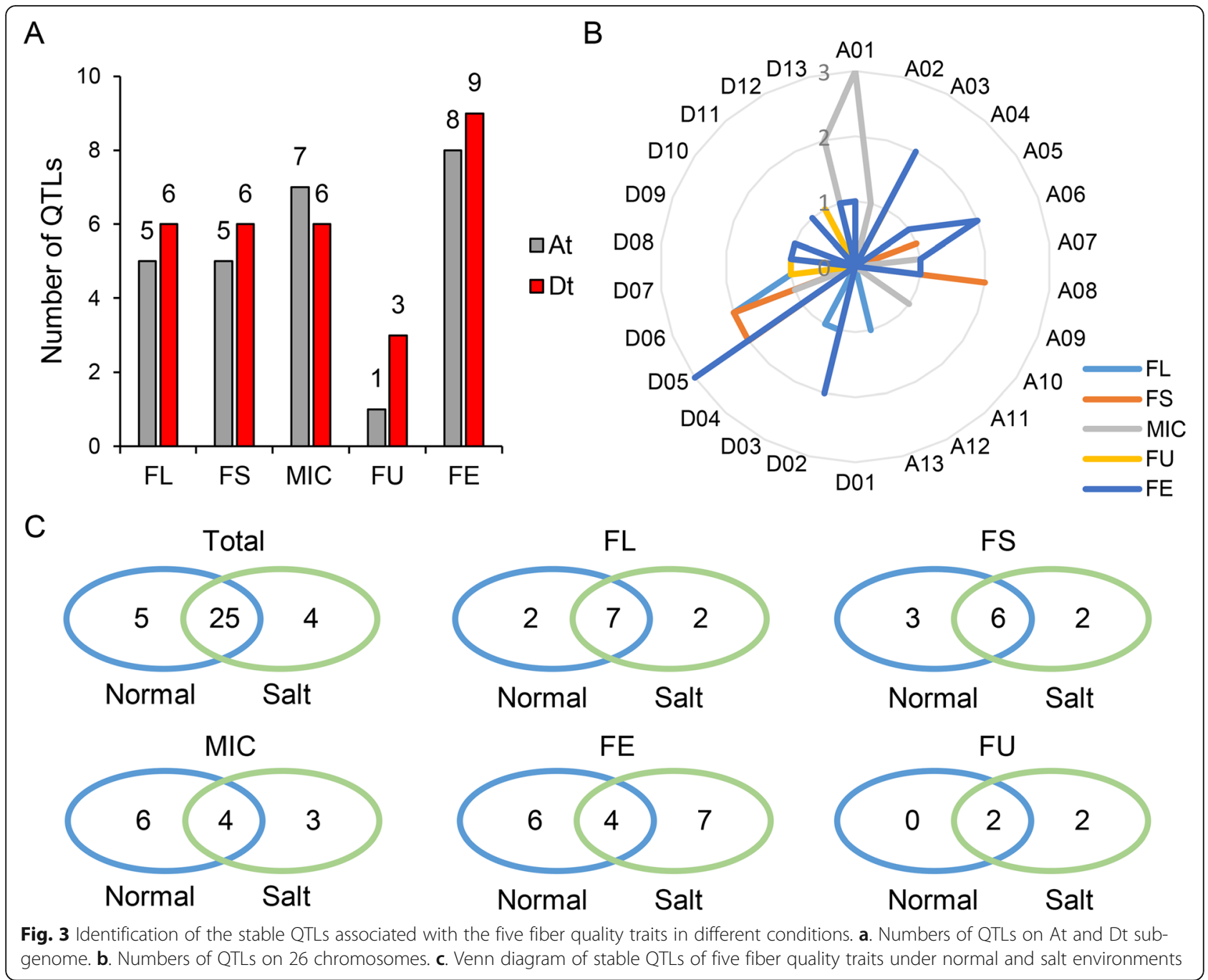

another candidate gene, GB_A03G0342 which encodes a laccase 3, was also identified. Laccase enzymes play important roles in cell elongation, lignification and pigmentation in plants and could play crucial role in cotton fiber quality [16]. Another high frequency associated QTL TM68443, which located on D08: 21,500,627 bp$21,900,627$ bp with SNP of A to G, was simultaneously associated with FL, FS and FU under the normal and salt environments. It showed that the FL, FS and FE increased by $8.1,26.9$ and $1.5 \%$ with the A haplotype comparing with the G haplotype, respectively (Additional file 13: Fig. S6). In the QTL region, a gene GB_D08G1034 encodes an auxin canalization protein, was identified. The previous study showed that auxin transport played an important role in fiber development of upland cotton [14]. In addition, we also found five high frequency QTLs only associated with a single trait, including one associated with FL, two associated with MIC, and two associated with FE. In these QTLs, many of the genes were related to fiber development, such as
GB_D09G2489 encodes a $\mathrm{Ca}^{2+}$-binding protein 1 [17] and GB_D09G2484 encodes a sodium hydrogen exchanger 2 [18], being important genes in fiber development under normal or salt environments.

\section{Discussion}

Upland cotton (G. hirsutum) and sea island cotton (G. barbadense) are two fiber-producing tetraploid cultivated cotton species, with markedly superior fiber quality in sea island cotton. However, there are few studies on the genetic characteristics of sea island cotton in natural population. In this study, we used high quality 6303 SNPs to investigate the genetic diversity and population characteristics of 279 sea island cotton accessions. Compared with the previous studies based on other molecular markers, such as ISSR [19], SRAP [20] and SSR [21], the number of SNP markers were greatly increased in present study. The mean PIC value of the 279 accessions showed that the PIC of At sub-genome was higher than that of Dt sub-genome. Phylogenetic tree and PCA 


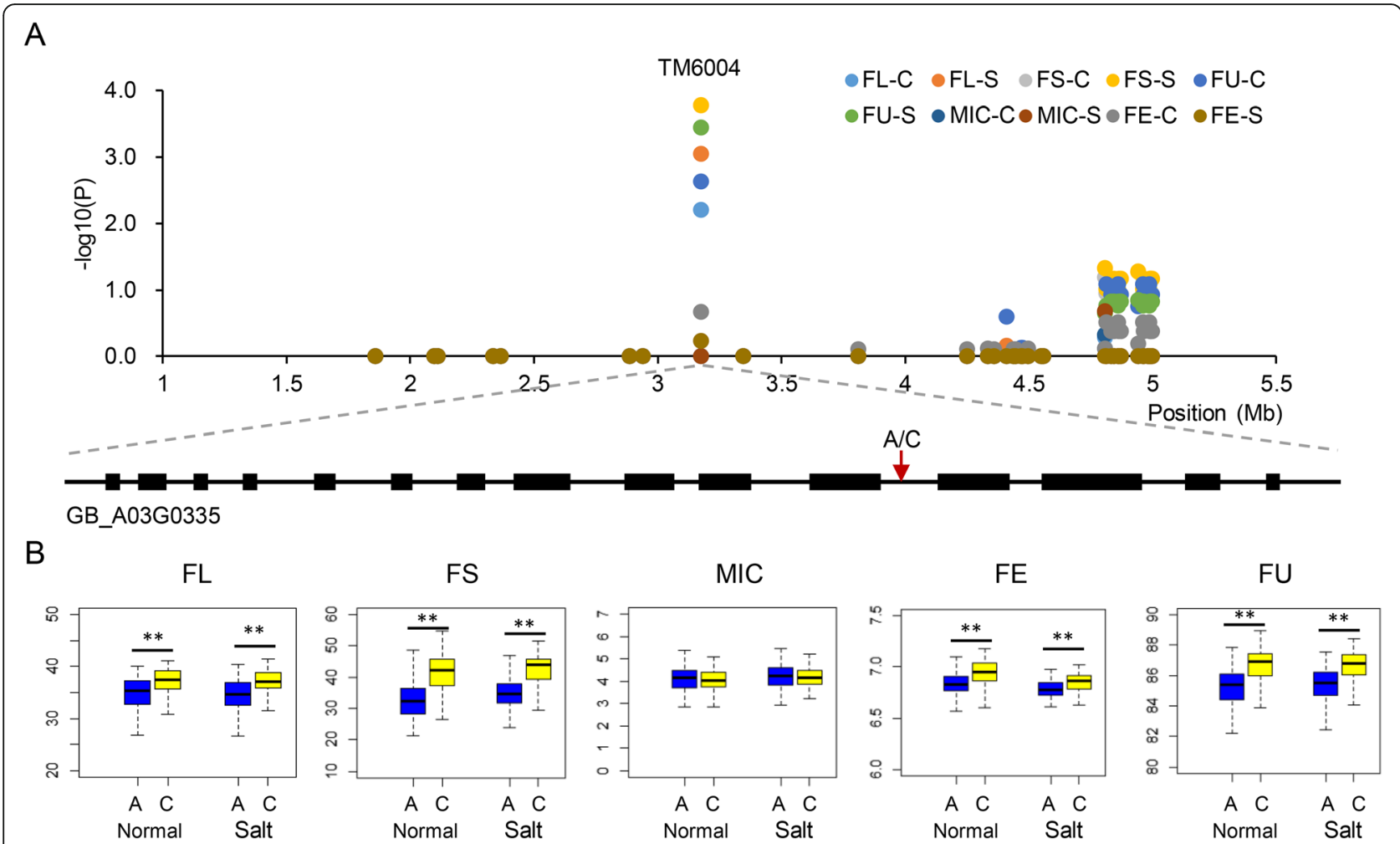

Fig. 4 Contribution of QTL TM6004 to fiber quality traits. a. Manhattan plots of SNPs around TM6004 for the best linear unbiased prediction (BLUP) of five traits. The location of a QTN for A/C related to GB_A03G0335. b. Box plots for the phenotypic values of QTN TM6004

analysis supported that the 279 accessions could be clustered into three groups, which was similar to previous studies based on SSR markers [21, 22]. Modern cultivated sea island cotton mainly includes three ecotypes: Egyptian type, American type and Middle-Asia type [22]. Of the 39 introduced landraces, Group1 contained three introduced accessions mainly from Egypt, of them, an original sea island cotton landrace, Menoufi, was also clustered in Group1. Group2 and Group3 were involved in widely introduced accessions from Egypt, USA and Former Soviet Union, with 31 in Group2 and five in Group3, implying that Group1 from original ecotype had the highest differentiation compared with the other two groups. At present, the genetic basis of modern cultivated sea island cotton is relatively narrow. Based on genetic diversity of three groups, the accessions from different groups can be crossed to pyramid multi-loci related to target traits for genetic improvement in future sea island cotton breeding.

Salinity is considered to be one of the most significant environmental factors limiting the growth and development of crop plants. In China, sea island cotton grows mainly in Xinjiang ecological area, however, most of the cultivated land is saline alkali in this region. Few studies were reported in stress condition for sea island cotton. Here, using sea island cotton natural population, we selected two different field environments, normal and saline alkali soil, to investigate systematically the fiber quality traits over 2 years. With phenotypic analysis, we found that FL and FE were significantly decreased in salt conditions, while FS and MIC were increased. In addition, no difference of FU was detected in the two environments. Of these changes, FS increased significantly in salt conditions with the largest variation compared with other four traits. High correlation among five fiber quality traits further supported the results. Consistent with the previous studies [11, 23], fiber length was decreased under salt stress, which required the sea island cotton varieties with improvement on fiber qualities, especially in fiber length, for planting in saline alkali condition.

Via GWAS analysis, a total of 34 stable QTLs, including nine high frequency associated loci, were identified to be related to five fiber quality traits. About half of these QTLs were associated with multiple traits, which was consistent with a high correlation among five fiber quality traits by phenotypic analysis. We found that four QTLs, TM27446_TM27440, TM33841_TM33859, TM58446 and TM68443, overlapped with the fiber quality traits associated QTLs of sea island cotton, reported recently by re-sequencing analysis [24]. Of them, TM68443 was identified as a high frequency associated 
QTL for FL, FS and FU in present study, and A haplotype of this QTL could significantly increase FL, FS and FE of sea island cotton. In addition, most QTLs, especially for FL and FS, were both detected in normal and salt conditions, indicating the high heritability of fiber quality traits in different environments. Besides these, we detected a fraction of QTLs associated with fiber quality specifically in salt environment and found the genes located in the QTLs were enriched in "response to stimulus", which might play important role for fiber improvement in saline alkali condition. We also found that the candidate genes simultaneously identified under normal and salt conditions were enriched in "xylem development", implying xylem participating in fiber development. Xylogen in the cell walls of cotton fibers effectively provides mechanical strength, while its functional role in fiber quality of sea island cotton remains to be explored.

To further mine the key genes affecting fiber qualities, we analyzed the high frequency QTLs detected more than 10 association times. Interestingly, seven of the total nine high frequency QTLs were detected both under normal and salt conditions. The QTL TM6004, which explained more than $21.6 \%$ of the variation in fiber quality traits. In this QTL region, the gene $G B_{-}$ A03G0335 which encodes a E3 ubiquitin-protein ligase, with a QTN variation from A to C in its intron region, showed that the FL, FS, FU and FE increased significantly by $6.9,22.5,1.5$ and $1.2 \%$ with the $C$ haplotype comparing with the A haplotype, respectively. Protein ubiquitination plays key roles in multiple plant developmental stages and several abiotic stress responses [25]. Ubiquitin-protein ligase has also reported to be involved in several biological processes in cotton. Overexpression an ubiquitin ligase gene, GhHUB2, increased fiber length and SCW thickness, while RNAi knockdown of GhHUB2 resulted in shortened fibers and thinner cell walls in cotton, which was involved in the ubiquitin-26S proteasome pathway [26]. The ubiquitin E3 ligase RHA2b promoted degradation of MYB30 [27], while MYB transcription factors were widely reported to be related to fiber development [28, 29]. Furthermore, E3 ubiquitin ligase was also reported to be involved in stress tolerance in cotton, such as Verticillium dahlia [30], drought [31] and salt tolerance [32]. Overexpression of a rice SUMO E3 ligase gene OsSIZ1 in cotton enhanced drought and heat tolerance, and substantially improved fiber yields in the field under reduced irrigation and rainfed farming conditions [33]. Based on transcription pattern in various vegetative and fiber development tissues in G. barbadense cv. Hai7124, we also found multiple types of transcripts of $G B \_A 03 G 0335$, implying that the gene GB_A03G0335 may play an important role in cotton fiber development and other stress tolerance.
However, the molecular mechanism remains to be confirmed in further study.

\section{Conclusions}

It is of great significance to explore the genetic characteristics and screen the key elite loci in sea island cotton for fiber quality improvement. In this study, we systematically reported genetic diversity of a set of sea island cotton natural population, and developed the phenotypic and GWAS analysis of five fiber quality traits under normal and salt environments. We found that the sea island cotton accessions were clustered into three groups with wide genetic diversity. There were high correlation of five fiber quality traits whether among traits in same environment or the same trait in different environments. Based on SNPs and GWAS analysis, a total of 34 stable QTLs were identified for the five fiber quality traits with 25 detected simultaneously under normal and salt environments. Haplotype analysis found that GB_A03G0335 encoding an E3 ubiquitin-protein ligase, play a crucial role in fiber quality and was significantly correlated with FL, FS, FU and FE. These findings will help us understand the genetic diversity of sea island cotton and contribute to cotton fiber quality improvement in breeding practice.

\section{Methods \\ Plant materials}

A total of 279 sea island cotton accessions, with 240 cultivars/lines collected from China and 39 introduced from abroad, were used for genotyping and population characteristic analysis, and 249 accessions were used for fiber phenotype analysis (Additional file 1: Table S1). Of these accessions, 11 landraces (E24-3389, Giza 36, Giza 45, L3398, Mi 10, Pima, Pima S-3, Termez 16, Yinzi 6022, 504-N, 9899И) were collected from the National Medium-term Gene Bank of Cotton in China, and other 268 accessions were collected and preserved by Nanjing Agricultural University and Xinjiang Agricultural University, China. All accessions were authorized for planting and investigating by Nanjing Agricultural University, Xinjiang Agricultural University and the National Medium-term Gene Bank of Cotton in China.

\section{Phenotype investigation and data analysis}

In 2017 and 2018, the 279 sea island cotton accessions were planted in two natural environments in Xinjiang Agricultural University Experimental Station. All necessary permits for the field evaluations of these accessions were authorized by Xinjiang Agricultural University, China. To assess soil salt concentration in the fields, soil samples were collected at $15-30 \mathrm{~cm}$ depth with nine sampling points for each environment before cotton planting, and were analyzed for electrical conductivity (EC) using the method described by Singh et al. (2017) 
[34]. The average soil EC was 0.5 and $2.7 \mathrm{dS} / \mathrm{m}$ in the two natural fields, and marked as normal and salt environments, respectively. Each accession was grown in two rows with a $3 \mathrm{~m}$ row length and $0.13 \mathrm{~m}$ between plants with two replicate plots in each environment. We collected 30 well developed open boll samples from the middle branches of each accession for fiber qualities detection. Totally, five fiber quality traits, FL, FS, MIC, FU and $\mathrm{FE}$, were measured using high volume fiber tester HFT9000 (ZXYQ09-2) by the Center of Cotton Fiber Quality Inspection and Testing, Ministry of Agriculture and Rural Affairs. Raw phenotypic data were also normalized across replicates, years, and environments using BLUP based on a mixed linear model in $\mathrm{R}$ package 'Ime4' [35]. Descriptive statistics of phenotype data are calculated through Excel software. Paired-samples $t$-tests and correlation analysis of phenotype data among different environments and traits were performed using SPSS software. Due to insufficient seedlings for phenotype investigation in 30 accessions, we used the phenotypic data of fiber quality traits from 249 accessions for further GWAS analysis.

\section{SNP genotyping}

For SNP genotyping, young leaves were collected from the 279 cotton accessions (a total of 288 samples, with eight collected repeatedly from different origins) and genomic DNA was extracted using CTAB method described by Paterson et al. (1993) [36]. A CottonSNP80K array containing 77,774 SNPs, was used to genotype the accessions. Qualified DNA was hybridized on the CottonSNP80K array and genotyped with the protocols described by Cai et al. (2017) [8]. The initial SNP data set was filtered with a calling rate $<0.8$ and $\mathrm{MAF}<0.01$. In addition, in order to determine the physical location of SNP on the reference genome, we mapped the probe sequences back to the Hai7124 genome [15] and the probe sequences mapped to multiple loci were further filtered. The finally obtained high quality data set was used for subsequent genetic and GWAS analysis.

\section{Population characteristics}

The SNP data format was adjusted by self-written shell script and used to conduct similarity analysis of 288 cotton accessions by PLINK V1.90 software [36]. For the construction of phylogenetic tree, a neighbor-joining method was used by Tassel 5.0 [37], and visually edited by FigTree software (http://tree.bio.ed.ac.uk/software/figtree/). Principal component analysis (PCA) was employed using Tassel 5.0 software and plot was drawn by R package "ggplot2". Admixture 1.3 software was applied to population structure analysis with $\mathrm{K}=3$ [38]. Nucleotide diversity $(\pi)$ and population divergence (Fst) between pairwise groups of sea island cotton accessions were calculated using Vcftools software [39].

\section{GWAS analysis}

The GWAS analysis was conducted using a multi-locus random-SNP-effect mixed linear model (mrMLM) [13] in R package "mrMLM". For the associated SNPs filtering in the first step of the model, the following parameters were set: Critical $P$-value in rMLM: 0.001; Search radius of candidate gene $(\mathrm{Kb}): 100$; and Critical LOD score in mrMLM: 3 . Meanwhile, the $\mathrm{Q}+\mathrm{K}$ model was also set with a population structure $(\mathrm{Q})$ matrix calculated by admixture 1.3 and a kinship (K) matrix calculated by R package "mrMLM". The Manhattan plot of associated QTN was performed by R software using the BLUP values of five traits in two environments.

\section{Identification of candidate genes}

We define the flank $200 \mathrm{~Kb}$ regions of QTN as the same QTL, and merge the overlapped QTLs to confirm the number of QTL. Putative candidate genes were identified in the QTL regions from the reference genome by self-written shell scripts. To determine the function of the candidate genes, Gene ontology (GO) analysis was implemented using AgriGO V2.0 with the SEA method [40]. The transcriptome profiles of various tissues and organs from Hai7124 were downloaded from NCBI Sequence Read Archive collection PRJNA490626 [15] and analyzed for mining candidate genes during fiber development. When FPKM (the fragments per kilobase of exon model per million mapped reads) of a gene was greater than 3 in ovules and fibers at $0-25$ day post anthesis (DPA), the gene was considered to be expressed during fiber development.

\section{Supplementary information}

Supplementary information accompanies this paper at https://doi.org/10. 1186/s12870-020-02502-4.

Additional file 1 Table S1. Information on 279 sea island cotton accessions used in this study.

Additional file 2 Figure S1. Call frequency distribution of total SNPS genotyped by CottonSNP80K array.

Additional file $\mathbf{3}$ Figure S2. Distribution of SNP number on each chromosome.

Additional file $\mathbf{4}$ Table S2. Information on high quality SNPS genotyped by CottonSNP80K array.

Additional file $\mathbf{5}$ Figure S3. Density distributions of five fiber quality traits in sea island cotton natural population.

Additional file 6 Figure S4. Correlation analysis of five fiber quality traits under different conditions. The number in these boxes indicated correlation coefficient (R value). ${ }^{*},{ }^{* *}$, and ${ }^{* * *}$ indicated $P$ value at the 0.05, 0.01 and 0.001 levels, respectively.

Additional file 7 Table S3. Stable QTLs of five fiber quality traits identified by multi-loci mixed linear model. 
Additional file 8 Figure S5. Distribution on detected times for 81 associated QTLs of five traits, respectively. The x-axis represents the detected times; $y$-axis represents the number of QTLS.

Additional file $\mathbf{9}$ Table S4. Information on the associated times and corresponding traits for stable QTLS.

Additional file $\mathbf{1 0}$ Table S5. Candidate genes associated with fiber quality traits within QTL regions.

Additional file 11 Table S6. GO analysis of genes specifically and simultaneously associated under normal and salt environments, respectively.

Additional file $\mathbf{1 2}$ Table S7. Expression profile of genes located in QTL regions related to fiber qualities.

Additional file $\mathbf{1 3}$ Figure S6. Box plots for the phenotypic values of QTN TM68443.

\section{Abbreviations}

BLUP: The best linear unbiased prediction; CV: Coefficients of variation; EC: Electrical conductivity; FE: Fiber elongation; FL: Fiber length; MIC: Fiber micronaire; FS: Fiber strength; FU: Fiber uniformity; GO: Gene Ontology; GWAS: Genome-wide association study; MAS: Marker assisted selection; MLM: Multi-loci mixed linear model; PIC: Polymorphism information content; QTLs: Quantitative trait loci; QTNs: Quantitative trait nucleotides; SLAFseq: Specific-locus amplified fragment sequencing; SNPS: Single nucleotide polymorphisms

\section{Acknowledgements}

We thank the high-performance computing platform of Bioinformatics Center in Nanjing Agricultural University for providing data analysis facilities. Many thanks to the National Medium-term Gene Bank of Cotton in China for providing 11 landraces (E24-3389, Giza 36, Giza 45, L-3398, Mi 10, Pima, Pima S-3, Termez 16, Yinzi 6022, 504-N, 9899h), and Nanjing Agricultural University and Xinjiang Agricultural University for providing other 268 sea island cotton accessions used in this study.

\section{Authors' contributions}

Experiments were designed by WZG. Experiments were performed by XJS, GZZ, XHS, HJX, WXL, XZN and QJC. GZZ and WZG drafted the manuscript, WZG revised the manuscript. All authors read and approved the final manuscript.

\section{Funding}

This program was financially supported in part by National Key R \& D Program for Crop Breeding (2018YFD0100400), National Natural Science Foundation in China (U1903203), the Fundamental Research Funds for the Central Universities (KYYJ201901), Jiangsu Collaborative Innovation Center for Modern Crop Production project (No.10), The funders were not involved in the experimental design of the study, data collection, analysis and interpretation, and in writing the manuscript.

\section{Availability of data and materials}

RNA-Seq data in this study have been deposited at the National Center of Biotechnology Information (NCBI, http://www.ncbi.nlm.nih.gov/) under the accessions PRJNA490626.

\section{Ethics approval and consent to participate}

Not applicable.

\section{Consent for publication}

Not applicable.

\section{Competing interests}

The authors declared that they had no competing interests.

\section{Author details}

'State Key Laboratory of Crop Genetics and Germplasm Enhancement, Engineering Research Center of Hybrid Cotton Development (the Ministry of Education), Nanjing Agricultural University, Nanjing 210095, China.

${ }^{2}$ Engineering Research Center for Cotton (the Ministry of Education), Xinjiang Agricultural University, Urumqi 830052, China. ${ }^{3}$ Institute of Industrial Crops,
Xinjiang Academy of Agricultural Sciences, Urumqi 830091, China. ${ }^{4}$ Cotton Research Institute, Xinjiang Academy of Agricultural and Reclamation Science, Shihezi 832000, China.

Received: 19 February 2020 Accepted: 17 June 2020

Published online: 22 June 2020

\section{References}

1. Cai C, Ye W, Zhang T, Guo W. Association analysis of fiber quality traits and exploration of elite alleles in upland cotton cultivars/accessions (Gossypium hirsutum L.). J Integr Plant Biol. 2014:56(1):51-62.

2. Shi Y, Li W, Li A, Ge R, Zhang B, Li J, Liu G, Li J, Liu A, Shang H, et al. Constructing a high-density linkage map for Gossypium hirsutum $\times$ Gossypium barbadense and identifying QTLs for lint percentage. J Integr Plant Biol. 2015:57(5):450-67.

3. Kohel RJ, Yu J, Park YH, Lazo GR. Molecular mapping and characterization of traits controlling fiber quality in cotton. Euphytica. 2001;121(2):163-72.

4. Fang L, Tian R, Li X, Chen J, Wang S, Wang P, Zhang T. Cotton fiber elongation network revealed by expression profiling of longer fiber lines introgressed with different Gossypium barbadense chromosome segments. BMC Genomics. 2014;15(1):838

5. Shen XL, Guo WZ, Zhu XF, Yuan YL, Yu JZ, Kohel RJ, Zhang TZ. Molecular mapping of QTLs for fiber qualities in three diverse lines in upland cotton using SSR markers. Mol Breed. 2005;15(2):169-81.

6. Sun Z, Wang X, Liu Z, Gu Q, Zhang Y, Li Z, Ke H, Yang J, Wu J, Wu L, et al. A genome-wide association study uncovers novel genomic regions and candidate genes of yield-related traits in upland cotton. Theor Appl Genet. 2018;131(11):2413-25.

7. Su J, Fan S, Li L, Wei H, Wang C, Wang H, Song M, Zhang C, Gu L, Zhao S, et al. Detection of favorable QTL alleles and candidate genes for lint percentage by GWAS in Chinese upland cotton. Front Plant Sci. 2016;7:1576.

8. Cai CP, Zhu GZ, Zhang TZ, Guo WZ. High-density $80 \mathrm{~K}$ SNP array is a powerful tool for genotyping G. hirsutum accessions and genome analysis. BMC Genomics. 2017;18(1):654

9. Sun Z, Li H, Zhang Y, Li Z, Ke H, Wu L, Zhang G, Wang X, Ma Z Identification of SNPs and candidate genes associated with salt tolerance at the seedling stage in cotton (Gossypium hirsutum L.). Front Plant Sci. 2018;9: 1011

10. Zhang ZY, Abuduwaili J, Yimit H. The occurrence, sources and spatial characteristics of soil salt and assessment of soil salinization risk in Yanq basin, Northwest China. PLoS ONE. 2014;9(9):e106079.

11. Sharif I, Aleem S, Farooq J, Rizwan M, Younas A, Sarwar G, Chohan SM. Salinity stress in cotton: effects, mechanism of tolerance and its management strategies. Physiol Mol Biol Plants. 2019:25(4):807-20.

12. Longenecker DE. The influence of high sodium in soils upon fruiting and shedding, boll characteristics, fiber properties, and yields of two cotton species. Soil Sci. 1974;118(6):387-96.

13. Wang SB, Feng JY, Ren WL, Huang B, Zhou L, Wen YJ, Zhang J, Dunwell JM, Xu S, Zhang YM. Improving power and accuracy of genome-wide association studies via a multi-locus mixed linear model methodology. Sci Rep. 2016;6:19444.

14. Zhu G, Gao W, Song X, Sun F, Hou S, Liu N, Huang Y, Zhang D, Ni Z, Chen $Q$, et al. Genome-wide association reveals genetic variation of lint yield components under salty field conditions in cotton (Gossypium hirsutum L.) BMC Plant Biol. 2020:20(1):23.

15. Hu Y, Chen JD, Fang L, Zhang ZY, Ma W, Niu YC, Ju LZ, Deng JQ, Zhao T, Lian JM, et al. Gossypium barbadense and Gossypium hirsutum genomes provide insights into the origin and evolution of allotetraploid cotton. Nat Genet. 2019:51(4):739-48.

16. Su J, Shim E, Noro J, Fu J, Wang Q, Kim HR, Silva C, Cavaco-Paulo A. Conductive cotton by in situ laccase-polymerization of aniline. Polymers. 2018;10(9):1023

17. Tang W, He Y, Tu L, Wang M, Li Y, Ruan YL, Zhang X. Down-regulating annexin gene GhAnn2 inhibits cotton fiber elongation and decreases $\mathrm{Ca}^{2+}$ influx at the cell apex. Plant Mol Biol. 2014;85(6):613-25.

18. He C, Yan J, Shen G, Fu L, Holaday AS, Auld D, Blumwald E, Zhang H Expression of an Arabidopsis vacuolar sodium/proton antiporter gene in cotton improves photosynthetic performance under salt conditions and increases fiber yield in the field. Plant Cell Physiol. 2005;46(11):1848-54.

19. Jiang $W$, Zhu HB, He JM. Genetic diversity in germplasm resources of cotton from different area based on ISSR markers. Cotton Sci. 2008;20(5):348-53. 
20. Li W, Ni W, Lin Z, Zhang X. Genetic diversity analysis of sea-island cotton cultivars using SRAP markers. Acta Agron Sin. 2008;34(5):893-8.

21. Wang $X Q$, Feng $C H$, Lin ZX, Zhang XL. Genetic diversity of sea-island cotton (Gossypium barbadense) revealed by mapped SSRs. Genet Mol Res. 2011;10(4):3620-31.

22. Ma Q, Zhao J, Lin H, Ning X, Liu P, Deng F, Si A, Li J. Association between SSR markers and fibre traits in sea island cotton (Gossypium barbadense) germplasm resources. J Genet. 2017;96(6):e55-63.

23. Peng J, Zhang L, Liu J, Luo J, Zhao X, Dong H, Ma Y, Sui N, Zhou Z, Meng Y. Effects of soil salinity on sucrose metabolism in cotton fiber. PLOS ONE. 2016;11(5):e0156398

24. Nie X, Wen T, Shao P, Tang B, Nuriman-Guli A, Yu Y, Du X, You C, Lin Z. High-density genetic variation maps reveal the correlation between asymmetric interspecific introgressions and improvement of agronomic traits in upland and pima cotton varieties developed in Xinjiang, China. Plant J. 2020. https://doi.org/10.1111/tpj.14760.

25. Shu K, Yang W. E3 ubiquitin ligases: ubiquitous actors in plant development and abiotic stress responses. Plant Cell Physiol. 2017;58(9):1461-76.

26. Feng H, Li X, Chen H, Deng J, Zhang CJ, Liu J, Wang T, Zhang XY, Dong JL. GhHUB2, a ubiquitin ligase, is involved in cotton fiber development via the ubiquitin-26S proteasome pathway. J Exp Bot. 2018;69(21):5059-75.

27. Zheng Y, Chen Z, Ma L, Liao C. The ubiquitin E3 ligase RHA2b promotes degradation of MYB30 in abscisic acid signaling. Plant Physiol. 2018;178(1):428-40.

28. Sun W, Gao Z, Wang J, Huang Y, Chen Y, Li J, Lv M, Wang J, Luo M, Zuo K. Cotton fiber elongation requires the transcription factor GhMYB212 to regulate sucrose transportation into expanding fibers. New Phytol. 2019; 222(2):864-81

29. Huang J, Guo Y, Sun Q, Zeng W, Li J, Li X, Xu W. Genome-wide identification of R2R3-MYB transcription factors regulating secondary cell wall thickening in cotton fiber development. Plant Cell Physiol. 2019;60(3):687-701.

30. Qin T, Liu S, Zhang Z, Sun L, He X, Lindsey K, Zhu L, Zhang X. GhCyP3 improves the resistance of cotton to Verticillium dahliae by inhibiting the E3 ubiquitin ligase activity of GhPUB17. Plant Mol Biol. 2019;99(4-5):379-93.

31. Chen H, Feng H, Zhang X, Zhang C, Wang T, Dong J. An Arabidopsis E3 ligase HUB2 increases histone $\mathrm{H} 2 \mathrm{~B}$ monoubiquitination and enhances drought tolerance in transgenic cotton. Plant Biotechnol J. 2019;17(3):556-68,

32. Liu Y, Zhang X, Zhu S, Zhang H, Li Y, Zhang T, Sun J. Overexpression of GhSARP1 encoding a E3 ligase from cotton reduce the tolerance to salt in transgenic Arabidopsis. Biochem Biophys Res Commun. 2016;478(4):1491-6.

33. Mishra N, Sun L, Zhu X, Smith J, Prakash Srivastava A, Yang X, Pehlivan N, Esmaeili N, Luo H, Shen G, et al. Overexpression of the rice SUMO E3 ligase gene OsSIZ1 in cotton enhances drought and heat tolerance, and substantially improves fiber yields in the field under reduced irrigation and rainfed conditions. Plant Cell Physiol. 2017;58(4):735-46.

34. Singh D, Singh CK, Kumari S, Singh Tomar RS, Karwa S, Singh R, Singh RB, Sarkar SK, Pal M. Discerning morpho-anatomical, physiological and molecular multiformity in cultivated and wild genotypes of lentil with reconciliation to salinity stress. PLoS ONE. 2017;12(5):e0177465.

35. Bates D, Machler M, Bolker BM, Walker SC. Fitting linear mixed-effects models using Ime4. J stat Softw. 2015; 67(1):1-48.36. Paterson, AH, Brubaker CL, Wendel JF. A rapid method for extraction of cotton (Gossypium spp.) genomic DNA suitable for RFLP or PCR analysis. Plant Mol Biol Rep. 1993; 11(2):122-7.

36. Purcell $S$, Neale B, Todd-Brown $K$, Thomas L, Ferreira MA, Bender D, Maller J, Sklar P, de Bakker PI, Daly MJ, et al. PLINK: a tool set for whole-genome association and population-based linkage analyses. Am J Hum Genet. 2007; 81(3):559-75.

37. Bradbury PJ, Zhang Z, Kroon DE, Casstevens TM, Ramdoss Y, Buckler ES. TASSEL: software for association mapping of complex traits in diverse samples. Bioinformatics. 2007;23(19):2633-5.

38. Alexander DH, Novembre J, Lange K. Fast model-based estimation of ancestry in unrelated individuals. Genome Res. 2009;19(9):1655-64.

39. Danecek P, Auton A, Abecasis G, Albers CA, Banks E, DePristo MA, Handsaker RE, Lunter G, Marth GT, Sherry ST, et al. The variant call format and VCFtools. Bioinformatics. 2011:27(15):2156-8

40. Tian T, Liu Y, Yan H, You Q, Yi X, Du Z, Xu W, Su Z. AgriGO v2.0: a GO analysis toolkit for the agricultural community, 2017 update. Nucleic Acids Res. 2017;45(W1):W122-9.

\section{Publisher's Note}

Springer Nature remains neutral with regard to jurisdictional claims in published maps and institutional affiliations.

Ready to submit your research? Choose BMC and benefit from:

- fast, convenient online submission

- thorough peer review by experienced researchers in your field

- rapid publication on acceptance

- support for research data, including large and complex data types

- gold Open Access which fosters wider collaboration and increased citations

- maximum visibility for your research: over $100 \mathrm{M}$ website views per year

At BMC, research is always in progress.

Learn more biomedcentral.com/submissions 